\title{
BMJ Open Protecting the healthcare workforce during COVID-19: a qualitative needs assessment of employee occupational health in the US national Veterans Health Administration
}

\author{
Cati Brown-Johnson (D) , ${ }^{1,2}$ Matthew D McCaa, ${ }^{1}$ Susan Giannitrapani, ${ }^{3}$ \\ Sara J Singer, ${ }^{1,2}$ Karl A Lorenz, ${ }^{1,2}$ Elizabeth M Yano, ${ }^{4,5,6}$ Wendy T Thanassi, ${ }^{2,7}$ \\ Cheyenne DeShields, ${ }^{1,8}$ Karleen F Giannitrapani (D) ${ }^{1,2}$
}

To cite: Brown-Johnson C, McCaa MD, Giannitrapani S, et al. Protecting the healthcare workforce during COVID-19: a qualitative needs assessment of employee occupational health in the US national Veterans Health Administration. BMJ Open 2021;11:e049134. doi:10.1136/ bmjopen-2021-049134

- Prepublication history and additional supplemental material for this paper are available online. To view these files, please visit the journal online (http://dx.doi.org/10.1136/ bmjopen-2021-049134).

Received 15 January 2021 Accepted 17 September 2021

Check for updates

(C) Author(s) (or their employer(s)) 2021. Re-use permitted under CC BY-NC. No commercial re-use. See rights and permissions. Published by BMJ.

For numbered affiliations see end of article.

Correspondence to Dr Karleen F Giannitrapani; karleen@stanford.edu

\section{ABSTRACT}

Objective Early in the COVID-19 pandemic, US Veterans Health Administration (VHA) employee occupational health $(\mathrm{EOH})$ providers were tasked with assuming a central role in coordinating employee COVID-19 screening and clearance for duty, representing entirely novel $\mathrm{EOH}$ responsibilities. In a rapid qualitative needs assessment, we aimed to identify learnings from the field to support the vastly expanding role of $\mathrm{EOH}$ providers in a national healthcare system.

Methods We employed rapid qualitative analysis of key informant interviews in a maximal variation sample on the parameters of job type, rural versus urban and provider gender. We interviewed $21 \mathrm{VHA} \mathrm{EOH} \mathrm{providers} \mathrm{between}$ July and December 2020. This sample represents 15 facilities from diverse regions of the USA (large, medium and small facilities in the Mid-Atlantic; medium sites in the South; large facilities in the West and Pacific Northwest).

Results Five interdependent needs included: (1) infrastructure to support employee population management, including tools that facilitate infection control measures such as contact tracing (eg, employeefacing electronic health records and coordinated databases); (2) mechanisms for information sharing across settings (eg, VHA listserv), especially for changing policy and protocols; (3) sufficiently resourced staffing using detailing to align $\mathrm{EOH}$ needs with human resource capital; (4) connected and resourced local and national leaders; and (5) strategies to support healthcare worker mental health.

Our identified facilitators for $\mathrm{EOH}$ assuming new challenging and dynamically changing roles during COVID-19 included: (A) training or access to expertise; (B) existing mechanisms for information sharing; (C) flexible and responsive staffing; and (D) leveraging other institutional expertise not previously affiliated with $\mathrm{EOH}$ (eg, chaplains to support bereavement).

Conclusions Our needs assessment highlights local and system level barriers and facilitators of $\mathrm{EOH}$ assuming expanded roles during COVID-19. Integrating changes both within and across systems and with alignment of human capital will enable $\mathrm{EOH}$ preparedness for future challenges.

\section{STRENGTHS AND LIMITATIONS OF THIS STUDY}

$\Rightarrow$ This is one of the first studies to evaluate the expanding role of employee occupational health $(\mathrm{EOH})$ in response to the COVID-19 pandemic.

$\Rightarrow$ The results of this study will help scale the dynamically changing job demands of $\mathrm{EOH}$, improving preparedness in advance of future pandemics.

$\Rightarrow$ Our analysis reveals needs of frontline $\mathrm{EOH}$ employees to keep healthcare workers (HCWs) safe from COVID-19 as an occupational hazard. Ensuring the safety of HCWs will help ensure the safety of the community at large.

$\Rightarrow$ These lessons are generalisable both beyond the Veterans Health Administration and beyond COVID-19.

$\Rightarrow$ Due to the condensed timeline, we used rapid analytic techniques, which should surface similar themes to in-depth coding but may not reveal deeper theoretical constructs.

\section{INTRODUCTION}

In the USA, healthcare workers (HCWs) were heavily burdened by COVID-19 due to increased frontline demands and increased exposure to the COVID-19, at times representing up to $20 \%$ of cases reported statewide. $^{1}{ }_{2}$ Beyond serious illness, HCWs were overworked during pandemic surges with worst case impacts extending even to suicide. ${ }^{34}$ While every aspect of healthcare delivery was impacted by the COVID-19 pandemic, supporting and protecting HCWs from COVID-19 as an occupational hazard must be of paramount ongoing importance, particularly as COVID-19 evolves, and we manage other future pandemics.

Given the heightened vulnerability of HCWs during the pandemic, employee occupational health $(\mathrm{EOH})$ providers were crucial 
in ensuring the safety of HCWs and thus the continuous delivery of healthcare. As of 2021, the US Veterans Health Administration (VHA) EOH assumes responsibility for the 'safety and health' of over a half million HCWs, trainees and volunteers. ${ }^{5}$ Representing a national healthcare system, the VHA serves over 9 million veterans, with 10000 in VHA community living center (CLC) nursing homes vulnerable to COVID-19. ${ }^{6}$ Furthermore, VHA comprises 1255 healthcare facilities and employs at least 322030 full-time HCWs ${ }^{5}$ the majority of whom fall in the US Occupational Safety and Health Administration (OSHA) very high risk category for SARS-CoV-2 transmission. ${ }^{7}$ VHA additionally interfaces with more than 73000 active volunteers, 15000 academic faculty and 127000 medical trainees. ${ }^{5}$

While VHA EOH has always been responsible for protecting this breadth of employees from workplace hazards, the COVID-19 pandemic required EOH to assume novel roles in managing the spread of infectious disease and to adapt as COVID-19 guidelines rapidly changed. On 15 March 2020, the US Deputy Under Secretary for Health for Operations and Management circulated guidance allowing asymptomatic HCWs exposed to COVID-19 to continue to work after consulting EOH and requiring HCWs to report to $\mathrm{EOH}$ if symptoms appeared at work, tasking EOH with a central role in COVID-19 management. ${ }^{8}$ After that announcement, VHA EOH policies surrounding COVID-19 continuously evolved; online media and VHA forums suggest frontline clinicians struggled to keep up with emerging COVID-19 recommendations. ${ }^{9}$ Other challenges stemmed from national personal protective equipment (PPE) shortages, which resulted in social media cries from HCWs to '\#GetMePPE' ${ }^{9}$ Similarly, VHA EOH was not consistently equipped with appropriate PPE at nationwide facilities, ${ }^{10}$ creating even more difficulties for $\mathrm{EOH}$ to fulfil new roles.

Our study leverages the perspectives of $\mathrm{EOH}$ to assess the barriers to and facilitators of $\mathrm{EOH}$ role expansion on the frontlines of supporting HCWs. In seeking to understand how best to support their expanding role, recent EOH publications on COVID-19 have relied on expert opinion $^{11}$ and literature review, ${ }^{12}$ as well as a growing number of qualitative reports. ${ }^{13}{ }^{14}$ Major themes from the literature include potential negative impacts of employee anxiety about COVID-19 $9^{15}$ and downstream impacts of telework such as social isolation or physical/ergonomic issues. ${ }^{12} \mathrm{EOH}$ healthcare literature reinforces these more general predictions of anxiety (especially related to burn-out) and has additionally highlighted risk factors surrounding overwork (eg, documenting requirements for electronic health records - EHR) and the potential protective impact of positive leadership. ${ }^{16}$

We undertook a rapid needs assessment for $\mathrm{EOH}$ as it assumed new and dynamically changing roles during COVID-19. Understanding needs and facilitating role readiness continues to be particularly critical as understanding about COVID-19 changes, guidance evolves and $\mathrm{EOH}$ prepares for future healthcare disasters.
Table 1 Respondent and site characteristics

\begin{tabular}{lr} 
Providers ( $\mathbf{n} \mathbf{2 1})$ & \\
Type & 10 \\
MD/DO & 8 \\
\hline NP/PA & 3 \\
\hline RN & \\
\hline Gender & 14 \\
\hline Women & 7 \\
\hline Men & \\
\hline Site (n=15) & \\
\hline Location & 5 \\
\hline Northeast & 3 \\
\hline Mid-Atlantic & 2 \\
\hline Midwest & 1 \\
\hline South & 1 \\
\hline Southwest & 3 \\
\hline West & \\
\hline Size & 6 \\
\hline Small & 3 \\
\hline Mid & 6 \\
\hline Large & \\
\hline Rural/urban & 4 \\
\hline Rural & \\
\hline Urban & \\
\hline
\end{tabular}

\section{METHODS}

Approach: We conducted 21 key informant qualitative interviews with $\mathrm{EOH}$ providers using a purposive sampling approach ${ }^{17}$ seeking variation on the parameters of provider type (lead providers: $\mathrm{MD} / \mathrm{DO}$, mid-level providers: NP/PA, RNs), setting (size, rural/urban and geographic region) and provider gender to represent a wide experience of $\mathrm{EOH}$ from this national health system (see table 1).

Our qualitative research team (CB, MM and $\mathrm{KG}$ ) developed the interview guide with input from two EOH subject matter experts (WT: physician, SG: nurse practitioner). The research advisory team (SS, KL and $\mathrm{EY}$ ) reviewed interview questions and procedures. The interview protocol addressed factors that could support or undermine readiness of EOH providers for COVID-19 expanded roles, notably documentation, reporting, staffing, etc (see online supplemental appendix A for interview protocol). In our purposive sample, we used a snowball approach ${ }^{17}$ starting with subject matter experts and then recruited with attention to sample variation in order to capitalise on diverse perspectives.

We sent potential participants an email including a study information sheet inviting them to interview, followed up by email twice and scheduled interviews with email respondents. During the phone interviews conducted by $\mathrm{PhD}$ trained qualitative research scientist (CB), investigators (CB and MM) obtained consent for audio recording. We 
captured notes during interviews for rapid analysis and created verbatim transcripts from audio recordings.

Analysis: We used standard qualitative methods, including rapid content analysis ${ }^{18} 19$ and member checking. ${ }^{20}$ Within the VHA, rapid qualitative approaches have successfully been used to provide real-time insights backed by highquality research methods. ${ }^{19}$ Indeed, a VHA comparison of rapid versus in-depth qualitative methods found the analyses to be consistent. ${ }^{21}$

Step 1: templated case summaries and team debriefing discussion to create initial themes. Per rapid qualitative analysis methods, we created templated case summaries for each interview, which were reviewed by the following team members $(\mathrm{CB}$ andKG and discussed during weekly debriefing calls with the research team. ${ }^{18}$ Initial themes were derived from these templated case summaries and debriefing calls (conducted with entire coauthor research team).

Step 2: creation and circulation of interim report for feedback (Lightning Report and modified member check). We used a Lightning Report approach-a rapid qualitative actionable product meant for wide distribution ${ }^{18}$ - to create a preliminary report based on themes from case summaries and postinterview debriefing calls once we had collected half of the data sample ( 2 months from first interview: $n=10$; see online supplemental appendix B for interim report). We circulated this Lightning Report to study advisors, VHA $\mathrm{EOH}$ central leadership and participants for feedback, constituting a modified synthesised member check. ${ }^{20}$

Step 3: integration of member check feedback and additional interviews for thematic saturation. Informed by EOH leader feedback and step 1's templated case summaries and team debriefing for the additional subsequent interviews $(n=11), C B$ and $K G$ formalised a provisional final theme list. This theme list was reviewed with research advisors and subject matter experts and iteratively modified to five themes representing needs with theme definitions and examples. Mental health needs emerged as a new theme in step 3, originating from templated case studies of the additional interviews.

Step 4: verification/query of themes with final transcripts. $\mathrm{CD}$ transcribed interviews and identified exemplary quotes from transcripts to represent the major themes. CB also reviewed transcriptions to confirm/disconfirm emergent themes.

\section{RESULTS}

We invited 95 potential participants and conducted 21 interviews with $\mathrm{EOH}$ providers (response rate 22\%). Interviews with $\mathrm{MD} / \mathrm{DO}(\mathrm{n}=10), \mathrm{NP} / \mathrm{PA}(\mathrm{n}=8)$ and $\mathrm{RN}(\mathrm{n}=3)$ participants were 30-60 min between July and December 2020. This sample represented 15 diverse VHA facilities from varied regions of the country, specifically large $(>4000$ employees), medium (2000<4000 employees) and small (<2000 employees) facilities in the Mid-Atlantic and Northeast, medium and large sites in South and Southwest and large facilities in the West and Pacific Northwest (see table 1 for sample demographics).

We report needs in five themes (see table 2 for exemplary quotes of each theme) organised around facilitators and barriers. Needs occurred within both systems and people. Systems needs included: (1) infrastructure to support population management locally and nationally and (2) mechanisms for information-sharing across the national system. People/human resources needs included: (3) sufficiently resourced staffing through detailing at the local level and (4) connected and resourced local and national leaders. A final theme around (5) mental health needs crossed both systems and people domains.

\section{Theme 1: infrastructure to support employee population management}

Across sites, respondents mentioned system needs locally and nationally (ie, at both the micro and macro levels) around population/infection management and tracking. Population health management needs revolved around tools and mechanisms that could prioritise employee privacy while facilitating infection control, for instance the need for employee-facing EHR or coordinated spreadsheet databases to support contact tracing. Infrastructure facilitators were tools (eg, EHR) and previous experience; barriers were lack of tools and lack of communication/ coordination.

\section{Facilitators}

Facilitators for population management included creation of tools and previous $\mathrm{EOH}$ provider experience with database management (eg, influenza vaccination) and infectious disease outbreaks. At a local (micro) level, independent VHA facilities created Excel spreadsheets to track employee testing; these in-house solutions were important for reporting the volume of employees served. In October 2020, VHA instituted an employee EHR at the national (macro) level, but some $\mathrm{EOH}$ providers perceived it as 'too little, too late... lack of cohesive connective EHR keeps people from optimal clinical care' (MD). In contrast, facilitators included previous experience with infectious disease outbreaks, training in public health management and incident command system training. One site reporting successful tracking and management shared that the 'first thing I did was make a centralised database... [but this was] a personal clinical decision' (RN).

\section{Barriers}

Barriers for population management included local lack of EHR for $\mathrm{EOH}$ as well as previous national cutbacks to EOH tracking. Lack of communication at some sites was also a barrier.

A major barrier for supporting population management was the lack of electronic health record (EHR) tools. However, EOH providers suggested employee population management infrastructure needs that encompassed much more than EHR. These needs also 


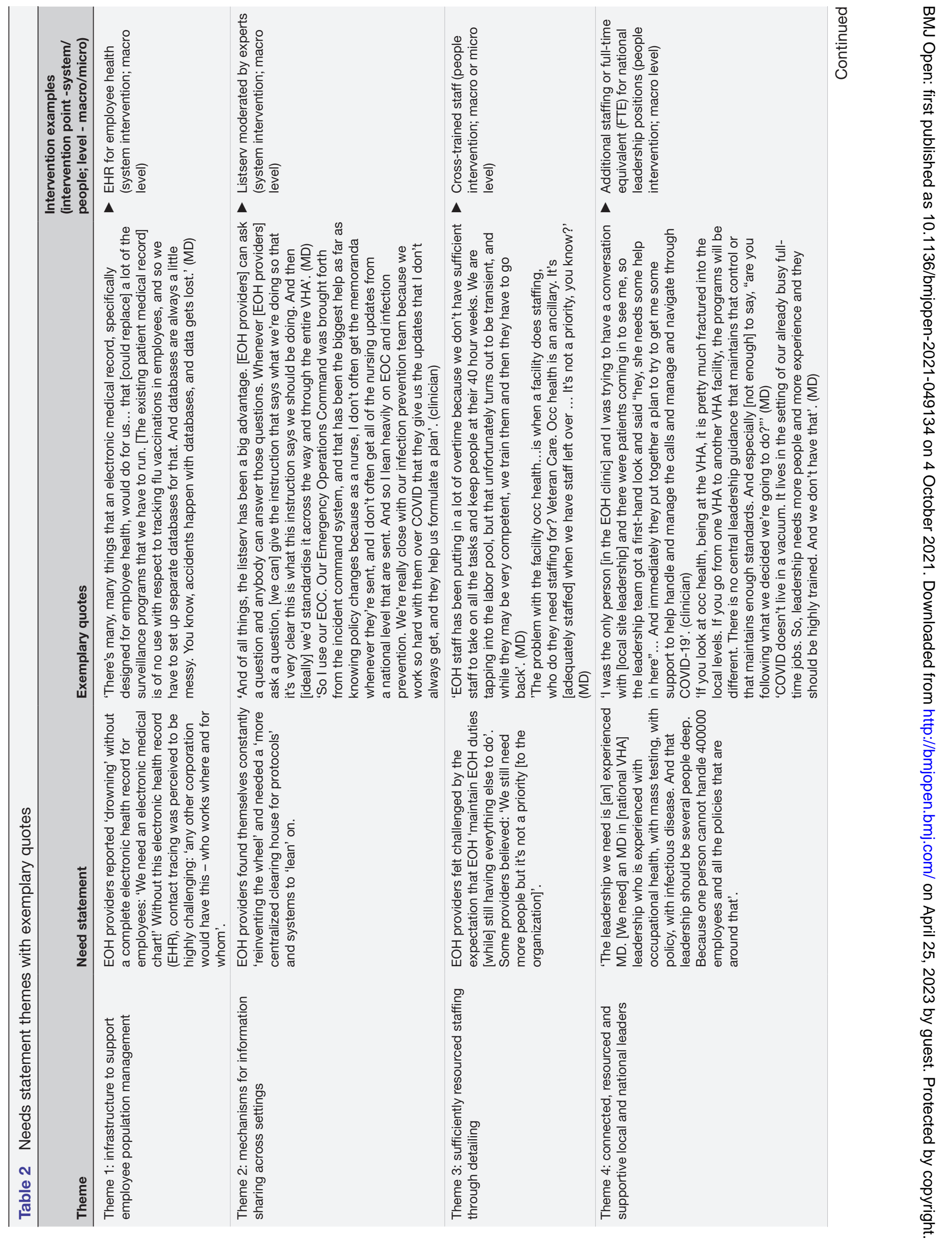




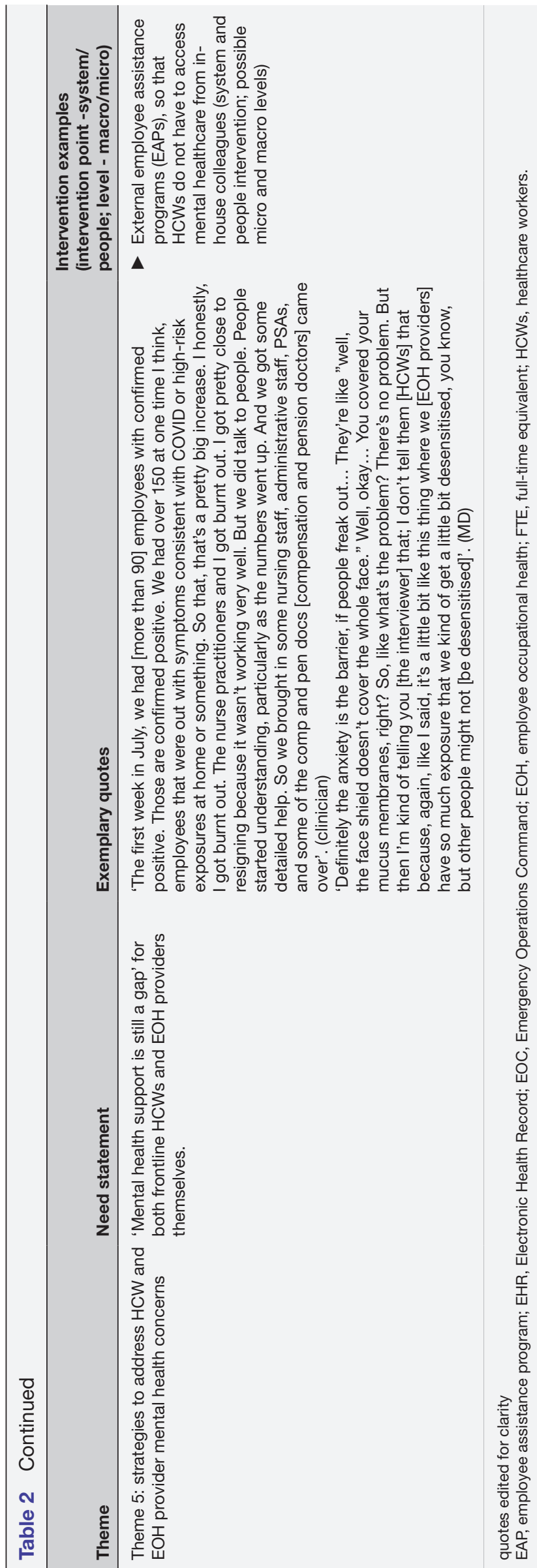

include: additional clinical space that could adequately address or limit cross-contamination for persons under investigation; support opportunities for innovation such as the use of QR code readers for testing and COVID-19 vaccination; and integrated backend infrastructure with worker compensation programs. Participants also cited lack of resources and recent cut-backs in EOH as major barriers to better population management. For instance, the EHR instituted in October was a new version of an EHR system that had existed some years earlier as version ' 1.0 ' but had been dropped during a budget cut.

Finally, lack of communication/consultation was also a barrier at some sites for population management. Locally, EOH requested being consulted when sites set up new systems to manage COVID-19. In one worst-case scenario, no one consulted EOH in the set-up of summer outside COVID-19 testing. As a result, "no one did risk assessment for heat stroke [in a parking lot] and there were no measures for shade... [They were] testing patients in plastic lawn chairs - unsafe for employees' (clinician).

Theme 2: mechanisms for information sharing across settings Providers reflected a strong need for information sharing within and across VHA facilities. Facilitators to information flow included access to external information sources and experts, as well as an existing all-VHA-EOH listserv. Barriers to information sharing included the unmoderated status of the listserv and the high volume of new information.

\section{Facilitators}

Facilitators for information included external information sources, such as the US Centers for Disease Control and Prevention (CDC) website, and even more broadly the internet, which supported information flow: '.. how I learned more and [tracked] the movement of the pandemic... [I] went to bed reading the CDC' (NP). Outside of state-sponsored information channels, strong connections with academic medicine facilitated information sharing. Providers reported benefitting from 'daily huddles with [academic infectious disease providers] when [the] knowledge base [was] exploding' (MD). A minority of providers reported closely reading and reviewing the VHA's Guidebook for Employee Health, which is 600 pages, but there was evidence that this resource was underused: 'questions on [the listserv] show that people don't use the Guidebook' (MD).

A listserv accessible to all VHA EOH was a major facilitator for information sharing. Many saw this peer-led listserv to be 'a big advantage' (RN). Providers reported the listserv, if adequately moderated by allocated experts, could support information sharing: 'ask a question [on the listserv], [experts] give the instruction... This is what we should be doing' (MD). Even in its unmoderated state in 2020, without the listserv some reported, 'we would all probably quit... [the listserv is] critical' (NP). 


\section{Barriers}

Barriers to information-sharing revolved around the extremely high volume of new information about COVID-19 and limitations of an unmoderated listserv. Due to the inexperience of temporary or untrained staff, the listserv could be perceived by more senior providers as 'extremely frustrating... every two weeks someone is asking that [same] question [due to] revolving door [staffing]' (MD). Indeed, some providers reflected a broader sense of discohesive information-sharing due to the listserv: 'Questions running rampant on the forum... there's no control' (NP).

\section{Theme 3: sufficiently resourced staffing through detailing and cross-training}

Not surprisingly, EOH providers reported that people, time and skills were needed to adequately resource $\mathrm{EOH}$ (eg, sufficient $\mathrm{EOH}$ employee full-time equivalent, or FTE, per HCW population) in the local site microenvironment. Alignment of human resource capital with $\mathrm{EOH}$ workforce needs was reported to facilitate new role requirements and protect the $\mathrm{EOH}$ workforce; lack of trained and consistent staff locally was a major barrier.

\section{Facilitators}

Additional staffing facilitators included creating standard EOH staffing ratios per employee (FTE), coverage/ cross-training for flexible scale-up and scale-down and alignment with services who could cover or be detailed to EOH when needed. Furthermore, even with adequate people on hand, 'the biggest thing we wanted... is cross-train [ing]' in areas vital to population health: call center management, testing, follow-up, and positive case management $(\mathrm{RN})$.

\section{Barriers}

For many EOH providers, a principle barrier to fulfilling EOH's new responsibilities was lack of staffing. Providers reported inconsistent staffing during the COVID-19 crisis: 'they would give us staff for only certain days and certain times'. Additionally, staffing needs doubled or tripled during surges, but numerous sites reported that training was lacking: these 'temporary folks who were detailed [were] slowly being pulled back into their own units' (NP), representing a major risk as the US met the 2020-21 winter COVID-19 surge. EOH providers wanted to be part of the conversation about staffing needs as they felt their site VHA executives might not always comprehend the scope of their expanded role or demands on their time.

EOH providers were also put in the position of managing employees' fear of COVID-19. Additional staffing was one strategy used to manage this employee anxiety. Multiple providers reported staff coming in early, staying late and working weekends to return calls: 'I put myself in their position. How would I feel [with no information]... My job is to protect them' (NP). Another provider ratified spending extra hours at work to return calls, 'People get so scared' (NP).

\section{Theme 4: connected and resourced local and national EOH leaders}

Providers emphasised the importance of having coherent guidance from national EOH leaders and interdisciplinary facility level executives. Successes at the local level were perceived as facilitated by interdisciplinary connections and inclusion in 'incident command'. Lack of resources in national leadership was seen as a barrier.

\section{Facilitators}

Local leaders (at the micro level) who were well networked were able to connect with crisis response 'incident command' structures, facilitating better EOH support for HCWs. These incident command structures generally included site leaders and daily meetings/huddles within EOH. COVID-19 teams such as this were appropriately reported as focusing on the 'veterans' perspective' (NP). EOH providers were perceived to be the 'only' role at this level of local leaders representing employee interests, needs and concerns. Providers perceived specific staff at national VHA EOH leadership to be 'excellent... extremely dedicated', but the positions were understaffed compared with the amount of work to be done: 'There is just one of them [1.0 FTE]' (MD). One recommended approach to effective centralised leadership included having two to three full-time experts who could 'travel to places that need experts... like consultants' (MD).

\section{Barriers}

By contrast, the perception of a barrier with respect to lack of adequate resources for leaders at the national level may have contributed to the sense that 'there isn't a coherent union of all [the VHA centers across the country]' (MD). VHA macro-level EOH leadership was perceived to need 'more staffing, more presence, structure that helps with outreach to all VAs... Boots on the ground' (MD). EOH providers wanted national level leaders to direct with authority during COVID-19, 'What you'd like is occupational health [central office leaders] coming out with rules to say "This is what we need to do" (MD).

\section{Theme 5: strategies to address HCW mental health concerns}

$\mathrm{EOH}$ providers, due to their role as a central point of contact with employees with a health-related workplace concern, found themselves in need of strategies to support HCW mental health during COVID-19. Both overwork and experiencing trauma (eg, excessive patient deaths or the death of a coworker) came up as examples which negatively impacted employee mental health. Outside of COVID-19 contagion, EOH providers recognised the impact on HCW mental health as the major impact of the pandemic on employee health: 'Anxiety is the barrier... Questions aren't just about work - "What about my toddlers and daycare and my 90-year-old grandmother?"” (MD). Incorporating external help (eg, employee 
assistance programs or non-EOH provider help including chaplains) was seen as a facilitator to supporting HCW mental health. Barriers to accessing mental health support related to the volume of HCW need and lack of local support for EOH. Additionally, EOH providers at multiple sites described themselves as on the brink of burnout due to exceptional and stressful workplace demands.

\section{Facilitators}

In one site, where nearly $50 \%$ of older patients had died in a surge, HCWs were grieving, distressed and bereaved. Facing the scale of this loss, local EOH leadership incorporated chaplain assistance in addition to referring HCW to employee assistance programs (EAPs). Looking to the future, one provider expressed that their $\mathrm{EOH}$ group knew 'to expect a tsunami of depression, anxiety, etc.' based on reading reports coming out of Japan, but this provider still did not have specific approaches to address this need locally (NP).

Some sites noted referring employees to EAPs for issues like 'tensions at home' but perceived that 'mental health support is still a [gap]' (MD). EOH attempted a wide range of strategies to support mental health for their employees, from referring HCWs to overwhelmed EAPs to system solutions such as facilitating easy access to VHA-issued laptops for employees to be able to work from home while on quarantine.

\section{Barriers}

EOH provider burnout and distress was a mental healthrelated barrier for better EOH care of HCWs. EOH providers consistently reported that they themselves were overwhelmed, and some reported nearing burnout. Multiple providers reported considering quitting-'I got pretty close to resigning' (MD) - due to the volume of work and positive cases. Furthermore, brittle VHA protocols not related to COVID-19 could plague EOH providers and contribute to burnout and distress. For example, in one instance, an $\mathrm{EOH}$ provider was repeatedly asked to justify overtime hours, even as their office was reduced to a single staff member managing $>3000$ employees.

\section{DISCUSSION}

Understanding how best to rapidly expand roles and scale the dynamically changing job demands of $\mathrm{EOH}$ during an infectious outbreak is needed in advance of future pandemics, and disaster preparedness is particularly important for this setting, the US VHA, which identifies preparedness as its 'Fourth Mission'. ${ }^{22}$ We took on this needs assessment when guidance in March 2020 from VHA national leadership forced EOH to the front and center of the organisation's response. ${ }^{8}$ We were particularly concerned that in order to minimise staffing shortages, healthcare organisations might choose to encourage potentially contagious but asymptomatic health personnel to work. Having EOH providers navigate this reality was complex, nuanced and something for which they had not prepared. EOH needed to learn, adapt and create new processes on the fly in a high-stakes setting.

Our identified facilitators of EOH assuming new challenging and dynamically changing roles during COVID-19 included: (A) training or access to expertise (in infectious diseases, public health management and disaster management); (B) existing mechanisms for information sharing (national reports from CDC and a VHA-specific listserv); (C) flexible and responsive staffing; and (D) leveraging other institutional expertise not previously affiliated with $\mathrm{EOH}$ (eg, chaplains to support mental health and bereavement).

In this qualitative systematic account of national $\mathrm{EOH}$ provider experiences, we found needs at the local and national level centered on systems/structure and people, similar to other international reports that identified preparedness, structures and physical/ mental health as primary challenges. ${ }^{14}$ Our study found primary barriers to $\mathrm{EOH}$ assuming expanded roles were related to funding for systems (eg, EHR implementation) and people, including limited staffing and leadership at both local and national levels.

In particular, the need for mental health and psychosocial support, identified in our fifth theme, has been documented to be a robust challenge for HCW internationally. ${ }^{13}{ }^{23-25}$ Other explorations of $\mathrm{EOH}$ needs during COVID-19 also identified EOH issues faced by medical health workers and overlapped with this study in terms of identifying work stressors and 'the need for supportive supervision' as major issues. ${ }^{26}$ Support for HCW mental health may be facilitated by organisational support and may underpin the psychological safety needed to nimbly respond to disasters. ${ }^{27}$ Rounding out the need for mental health support, recent reviews have identified frontline and non-physician HCWs as having the greatest mental health needs of HCWs during the COVID-19 pandemic. ${ }^{24}$

As COVID-19 persists and other pandemics emerge, the role of employee occupational health providers in national healthcare systems should not be undervalued. Though the role of EOH may be underestimated or unconsidered in healthcare settings, it is critical to the safety of the healthcare workforce. Furthermore, EOH's potential role in minimising COVID-19 spread among HCWs is directly relevant to the safety of employees and their families, vulnerable patients and the community at large.

\section{Promising practices beyond VHA}

Despite the fluctuation of recommendations from some national agencies (eg, $\mathrm{CDC}),{ }^{28}$ our $\mathrm{EOH}$ providers reported relying heavily on external agency standards to inform their local response. Ideally, national leadership could provide enough guidance that in times of crisis individual sites are not learning by themselves; intersite communication gave $\mathrm{EOH}$ providers a community to 
engage in shared learning and to accelerate spread of learnings, processes and policy adaptations. Thinking beyond acute disasters, a high-functioning national $\mathrm{EOH}$ community in an integrated healthcare system could even potentially positively address long-standing health and civil wellness issues (eg, racism and racial inequality).

\section{Promising practices beyond COVID-19}

These lessons from the COVID-19 pandemic critically inform future $\mathrm{EOH}$ preparedness. Past healthcare crises have pointed to the demand for decisive leadership, collaborative networks and employee monitoring systems, ${ }^{29}$ echoing the needs of VHA EOH. Although EOH providers felt the VHA piloted the employee EHR too late in response to COVID-19, this EHR system will likely prove useful in the future. In the wake of increasing epidemics and natural disasters, it is crucial that we recognise both the immediate and long-term benefits of equipping EOH with the tools to expand their role in managing HCW safety.

\section{Limitations}

To rapidly produce early insights for the field, we leveraged a rapid qualitative analytic approach instead of more in-depth qualitative methods. This approach optimised dissemination of frontline provider insights in preparation for the COVID-19 vaccine roll-out in December 2020. Previous reports have shown that rapid and in-depth qualitative analysis can produce the same results, ${ }^{21}$ but we may have missed important theoretical insights as a result of rapid analysis, which we hope to remedy with future in-depth theoretical analyses. We were ultimately able to produce early results in just 2 months, and some recommendations originating from our participants are already being enacted by the VHA. Secondarily, our purposive snowball sample is a good snapshot of the experiences of $\mathrm{EOH}$ in the VHA, but ideally we might have talked to $\mathrm{EOH}$ providers from each major site, since pandemic progression varied greatly from location to location, even within the USA.

\section{CONCLUSION}

In our highly networked world, EOH will consistently be at the forefront of disaster management and will continue to be central in future pandemics. A systematic focus on $\mathrm{EOH}$ in healthcare settings will be a strong step towards truly honouring the effort HCWs put forward in the COVID-19 pandemic, keeping them safe in their places of work. As one of our participants highlighted, 'Employees are the key asset, [but] without $[\mathrm{EOH}]$ occupational health professionals... we are not able to support and optimize the health of employees'.

\section{Author affiliations}

${ }^{1}$ Center for Innovation to Implementation, VA Palo Alto Health Care System, Menlo Park, California, USA
${ }^{2}$ Division of Primary Care and Population Health, Stanford University School of Medicine, Stanford, California, USA

${ }^{3}$ Department of Employee Occupational Health, Wilmington VA Medical Center, Wilmington, Delaware, USA

${ }^{4}$ Center for the Study of Healthcare Innovation, Implementation \& Policy, VA Greater Los Angeles Healthcare System, Los Angeles, California, USA

${ }^{5}$ Department of Health Policy and Management, University of California Los Angeles Fielding School of Public Health, Los Angeles, California, USA

${ }^{6}$ Department of Medicine, UCLA Geffen School of Medicine, Los Angeles, California, USA

${ }^{7}$ Occupational Health Service, VA Palo Alto Health Care System, Palo Alto, California, USA

${ }^{8}$ The Jack, Joseph, and Morton Mandel School of Applied Social Sciences, Case Western Reserve University, Cleveland, Ohio, USA

Acknowledgements We would like to thank all the providers who participated in interviews and our partnership with US Veterans Health Administration (VHA) occupational health leadership. Natalie Connell should be acknowledged for her help with manuscript preparation. The contents of this manuscript do not represent the views of VHA or the US Government. This study was reviewed and approved by the Stanford and VHA IRB, \#56617; participants gave informed consent before participating. This work was supported by COVID-19 Pilot Project Award \# C19 20-207 (Principal Investigator, K Giannitrapani) and Senior Research Career Scientist Award RCS 05-195 (Principal Investigator, Yano) from the US Department of Veterans Affairs, Health Services Research and Development Service. This material is the result of work supported with resources and the use of facilities at the VA Palo Alto Health Care System, Palo Alto, California. CB is at the Center for Innovation to Implementation, VA Palo Alto Healthcare System, Menlo Park, California. MM is at the Center for Innovation to Implementation, VA Palo Alto Healthcare System, Menlo Park, California. SG is in Employee Occupational Health, Wilmington VA Medical Center, Wilmington, Delaware. SS is at the Center for Innovation to Implementation, VA Palo Alto Healthcare System, Menlo Park, California. KL is at the Center for Innovation to Implementation, VA Palo Alto Healthcare System, Menlo Park, California. EY is at Center for the Study of Healthcare Innovation, Implementation \& Policy, VA Greater LA Healthcare System, Los Angeles, California. WT is with the Occupational Health Service, VA Palo Alto Healthcare System, Palo Alto, California. CD is at the Center for Innovation to Implementation, VA Palo Alto Healthcare System, Menlo Park, California. KG is a Core Investigator, Center for Innovation to Implementation, VA Palo Alto Healthcare System, Menlo Park, California.

Contributors KG, SG and CB were responsible for the conception and design of this study. Research advisors, KL, SS, and EY helped CB, KG, MM and CD design the interview protocol. SG and WT facilitated recruitment of EOH providers. CB and MM conducted data collection, and $\mathrm{CD}$ transcribed interviews and participated in analysis. $\mathrm{CB}$ and $\mathrm{KG}$ drafted the manuscript, with substantial input and review from all authors. All authors approved the final manuscript.

Funding VA HSR\&D C19 20-207 (PI K Giannitrapani); VA HSR\&D RCS 05-195 (PI Yano).

Competing interests WT and SG are VHA occupational health employees; they facilitated recruitment and commented on synthesised results.

Patient consent for publication Not applicable.

Provenance and peer review Not commissioned; externally peer reviewed.

Data availability statement Data are available on reasonable request. Deidentified data are available upon reasonable request.

Supplemental material This content has been supplied by the author(s). It has not been vetted by BMJ Publishing Group Limited (BMJ) and may not have been peer-reviewed. Any opinions or recommendations discussed are solely those of the author(s) and are not endorsed by BMJ. BMJ disclaims all liability and responsibility arising from any reliance placed on the content. Where the content includes any translated material, BMJ does not warrant the accuracy and reliability of the translations (including but not limited to local regulations, clinical guidelines, terminology, drug names and drug dosages), and is not responsible for any error and/or omissions arising from translation and adaptation or otherwise.

Open access This is an open access article distributed in accordance with the Creative Commons Attribution Non Commercial (CC BY-NC 4.0) license, which permits others to distribute, remix, adapt, build upon this work non-commercially, and license their derivative works on different terms, provided the original work is 
properly cited, appropriate credit is given, any changes made indicated, and the use is non-commercial. See: http://creativecommons.org/licenses/by-nc/4.0/.

\section{ORCID iDs}

Cati Brown-Johnson http://orcid.org/0000-0002-5415-3665

Karleen F Giannitrapani http://orcid.org/0000-0003-0987-6204

\section{REFERENCES}

1 Spencer J. Why we're tracking every US health worker who dies from coronavirus, 2020. Available: https://www.theguardian.com/us-news/ 2020/apr/15/why-were-tracking-every-us-health-worker-who-diesfrom-coronavirus [Accessed 4 Dec 2020].

2 Kambhampati AK. COVID-19-Associated hospitalizations among health care personnel. Morbidity and Mortality Weekly Report 2020;69.

3 Gulati G, Kelly BD. Physician suicide and the COVID-19 pandemic. Occup Med 2020.

4 Danhauer SC, Files K, Freischlag JA. Physician Suicide-Reflections on relevance and resilience. JAMA Surg 2020;155:721-2.

5 Veterans Health Administration. About VHA - Veterans Health Administration, 2019. Available: https://www.va.gov/health/aboutvha. asp [Accessed 4 Dec 2020].

6 U. S. Government Accountability Office. VA nursing home care: VA has opportunities to enhance its oversight and provide more comprehensive information on its website, 2019. Available: https:// www.gao.gov/products/GAO-19-428 [Accessed 14 Dec 2020].

7 Occupational Safety and Health Administration. Worker exposure risk to COVID-19, 2020. Available: https://www.osha.gov/coronavirus [Accessed 17 Dec 2020].

8 Liberman S. Coronavirus (COVID019) guidance for work recommendations for asymptomatic healthcare personnel after exposure to a COVID-19 patient U.S. Department of Veterans Affairs, Deputy Undersecretary for Health for Operations and Management; 2020.

9 Padilla M. 'It Feels Like a War Zone': Doctors and Nurses Plead for Masks on Social Media, 2020. The new York times. Available: https://www.nytimes.com/2020/03/19/us/hospitals-coronavirus-ppeshortage.html [Accessed 14 Dec 2020].

10 US Department of Veterans Affairs, Veterans Health Administration. Veterans health administration (VHA) coronavirus disease 2019 (COVID-19) response report, 2020. Available: https://www. publichealth.va.gov/n-coronavirus/ [Accessed 14 Dec 2020].

11 Sinclair RR, Allen T, Barber L, et al. Occupational health science in the time of COVID-19: now more than ever. Occup Health Sci 2020:1-22.

12 Bouziri H, Smith DRM, Descatha A, et al. Working from home in the time of COVID-19: how to best preserve occupational health? Occup Environ Med 2020;77:509-10.

13 Roca J, Canet-Vélez O, Cemeli T, et al. Experiences, emotional responses, and coping skills of nursing students as auxiliary health workers during the peak COVID-19 pandemic: a qualitative study. Int $\checkmark$ Ment Health Nurs 2021;30:1080-92.
14 Jeleff M, Traugott M, Jirovsky-Platter E. Occupational challenges of health care workers during the COVID-19 pandemic. A qualitative study. medRxiv2021.

15 Trougakos JP, Chawla N, McCarthy JM. Working in a pandemic: exploring the impact of COVID-19 health anxiety on work, family, and health outcomes. J Appl Psychol;105:1234-45.

16 Buselli R, Baldanzi S, Corsi M, et al. Psychological care of health workers during the COVID-19 outbreak in Italy: preliminary report of an occupational health department (AOUP) responsible for monitoring hospital staff condition. Sustainability 2020;12:5039.

17 Bernard HR. Research methods in anthropology: qualitative and quantitative approaches. 4th ed. Lanham, MD: AltaMira Press, 2006.

18 Brown-Johnson C, Safaeinili N, Zionts D, et al. The Stanford lightning report method: a comparison of rapid qualitative synthesis results across four implementation evaluations. Learn Health Syst 2020;4:e10210.

19 Hamilton AB, Brunner J, Cain C, et al. Engaging multilevel stakeholders in an implementation trial of evidence-based quality improvement in VA women's health primary care. Trans/ Behav Med 2017;7:478-85.

20 Birt L, Scott S, Cavers D, et al. Member checking: a tool to enhance Trustworthiness or merely a NOD to validation? Qual Health Res 2016;26:1802-11.

21 Gale RC, Wu J, Erhardt T, et al. Comparison of rapid vs in-depth qualitative analytic methods from a process evaluation of academic detailing in the Veterans health administration. Implement Sci 2019;14:11.

22 Massarweh NN, Itani KMF, Tsai TC. Maximizing the US department of Veterans Affairs' reserve role in national health care emergency Preparedness-The fourth mission. JAMA Surg 2020;155:913-4.

23 Chen $\mathrm{Q}$, Liang M, Li Y, et al. Mental health care for medical staff in China during the COVID-19 outbreak. Lancet Psychiatry 2020;7:e15-16.

24 Moitra M, Rahman M, Collins PY, et al. Mental health consequences for healthcare workers during the COVID-19 pandemic: a scoping review to draw lessons for LMICs. Front Psychiatry 2021;12:22.

25 Spoorthy MS, Pratapa SK, Mahant S. Mental health problems faced by healthcare workers due to the COVID-19 pandemic-A review. Asian J Psychiatr 2020;51:102119.

26 Krystal JH, Alvarado J, Ball SA, et al. Mobilizing an institutional supportive response for healthcare workers and other staff in the context of COVID-19: the Yale experience. Gen Hosp Psychiatry 2021;68:12-18.

27 Lee H. Changes in workplace practices during the COVID-19 pandemic: the roles of emotion, psychological safety and organisation support. Journal of Organizational Effectiveness: People and Performance 2021;8:97-128.

28 Benzian $\mathrm{H}$, Johnston M, Stauf N, et al. Presenting or spinning facts? Deconstructing the U.S. centers for disease control statement on the importance of reopening schools under COVID-19. Front Public Health 2021;9:645229.

29 Khan Y, O'Sullivan T, Brown A, et al. Public health emergency preparedness: a framework to promote resilience. BMC Public Health 2018;18:1344. 